\title{
Non-radioisotopic glucose turnover in children with falciparum malaria and enteric fever
}

Balbir Singh1, Keng Ee Choo', Jamal Ibrahim1, Wayne Johnston ${ }^{3}$ and Timothy M. E. Davis ${ }^{3}$

1 Department of Medical Microbiology and Parasitology, Universiti Sains Malaysia, Kubang Kerian, Kelantan, Malaysia; 2 Kota Bharu Hospital, Kelantan, Malaysia. 3 University of Western Australia, Department of Medicine, Fremantle Hospital, Fremantle, Australia

\begin{abstract}
To determine whether glucose turnover is increased in acute falciparum malaria compared to enteric fever in children, steady-state $6,6-\mathrm{D}_{2}$-glucose turnover was measured in 9 Malaysian children with uncomplicated malaria ( 6 males and 3 females; median age 10 years, body weight $22 \mathrm{~kg}$ ) and in 12 with uncomplicated enteric fever ( 8 males and 4 females; median age 10 years, body weight $24 \mathrm{~kg}$ ) in acute illness, after quinine ( 5 malaria patients) and in convalescence. Baseline plasma glucose concentrations in malaria and enteric fever were similar (all values are medians [ranges in brackets]) $5 \cdot 6$ [3.2-11.3] vs. $5 \cdot 5[4 \cdot 2-8 \cdot 0] \mathrm{mmol} / \mathrm{L})$, as were serum insulin levels $(5 \cdot 6[0 \cdot 4-26 \cdot 5] \mathrm{vs} .6 \cdot 8[1 \cdot 1-22 \cdot 5]$ milliunits $/ \mathrm{L}$; $P>0.4)$. Glucose turnover in the malaria patients was higher than in patients with enteric fever $(6 \cdot 27$ $[2 \cdot 71-6 \cdot 87]$ vs. $5 \cdot 20[4.50-6 \cdot 08] \mathrm{mg} / \mathrm{kg} . \mathrm{min} ; P=0 \cdot 02)$ and in convalescence $(4 \cdot 74[3 \cdot 35-6 \cdot 79] \mathrm{mg} /$ kg.min; $P=0.05$ vs. acute malaria study), and fell after quinine together with a rise in serum insulin $(P=0.03)$. Basal plasma lactate concentrations were higher in enteric fever than in malaria $(3 \cdot 4[1 \cdot 8-6 \cdot 4]$ vs. $0.8[0.3-3.8] \mathrm{mmol} / \mathrm{L} ; P<0.0001)$ and correlated inversely with glucose turnover in this group $\left(r_{\mathrm{s}}=-0.60 ; n=12 ; P=0.02\right)$. These data suggest that glucose turnover is $20 \%$ greater in malaria than in enteric fever. This might reflect increased non-insulin-mediated glucose uptake in falciparum malaria and/ or impaired gluconeogenesis in enteric fever, and may have implications for metabolic complications and their clinical management in both infections.
\end{abstract}

Keywords: malaria, Plasmodium falciparum, enteric fever, Salmonella typhi, glucose turnover, insulin, gluconeogenesis, quinine, children

\section{Introduction}

Hypoglycaemia is more than twice as likely to complicate falciparum malaria in children than in adults both before and during treatment (WHITE et al., 1987; MARSH et al., 1995; HIEN et al., 1996; VAN-HENSBROEK et al., 1996). However, there is still debate as to whether malaria is more commonly associated with hypoglycaemia than are other serious paediatric infections (KAWO et al., 1990). A central issue is the comparability of the clinical severity of falciparum malaria and other infections in children (BREWSTER et al., 1990; DAVIS, 1990; TAYLOR et al., 1990). One view is that hypoglycaemia is related to the time of the last meal and is correctable when identified (KAWO et al., 1990). By contrast, hypoglycaemia has been considered to be closely associatcd with spccific complications of malaria such as coma, when it may prove refractory to treatment and signify a poor prognosis (TAYLOR et al., 1990).

Glucose turnover is accelerated by approximately $50 \%$ in both untreated, non-pregnant adults with severe falciparum malaria (DAVIS et al.; 1993) and in pregnant women with uncomplicated Plasmodium falciparum or P. vivax malaria (DAvIS et al., 1994). Although control studies in healthy children remain ethically problematic, available evidence suggests that glucose turnover is, by contrast, decreased in children with uncomplicated falciparum malaria (DEKKER et al., 1996). This difference may reflect age-related changes in the balance between glucose production and peripheral utilization. A negative association between the plasma glucose concentration and its turnover in severely ill adults suggests that increased peripheral glucose uptake predominates (DAVIS et al., 1993; BINH et al., 1997). A positive correlation between plasma glucose and its turnover in children with uncomplicated malaria supports the view that hepatic glucose production is the main determinant of glycaemia in this age group (DEKKER et al., 1996)

Direct and indirect effects of the metabolically active $P$. falciparum parasite in the microvasculature of organs

Address for correspondence: Professor T. M. E. Davis, University of Western Australia, Department of Medicine, Fremantle Hospital, P.O. Box 480, Fremantle, Western Australia 6959 Australia; phone +61894313229, fax +61894312977, e-mail tdavis@cyllene.uwa.edu.au such as the liver (MACPHERSON et al., 1985) and treatment with quinine (DAVIS et al., 1990) may influence glucose metabolism in malaria and increase the likelihood of hypoglycaemia relative to its occurrence in other paediatric infections. To investigate this hypothesis in a stable clinical situation in which valid metabolic data could be collected, we measured glucose turnover using a safe, minimally recycled, non-radioactive glucose isotope $\left(6,6-\mathrm{D}_{2}\right.$-glucose $)$ in a group of Malaysian children with acute uncomplicated falciparum malaria. The same measurements were performed in a control group of children with enteric fever, a common bacterial infection in tropical countries. The results suggest that glucose production is relatively high in malaria due to increased non-insulin mediated glucose uptake in falciparum malaria and/or impaired gluconeogenic capacity in enteric fever.

\section{Patients and Methods}

\section{Patients}

We studied 9 children with acute uncomplicated falciparum malaria (WARRELL et al., 1990) and 12 with enteric fever (see Table 1). All had been admitted to Kuala Krai, Tanah Merah, Pasir Mas or Kota Bharu Hospitals, Kelantan State, Malaysia. All patients with malaria had blood films containing $P$. falciparum and those with enteric fever had blood or stool cultures with Salmonella typhi, and/or had a positive Widal test or dotenzyme immunosorbent assay (ISMAIL et al., 1991). None of the patients had other illnesses, including diarrhoeal disease, and none had clinical evidence of severe malnutrition or prolonged starvation before admission. Each patient's parent or guardian gave witnessed, informed consent to participation. The study protocol was approved by the ethics committee of the Ministry of Health, Malaysia.

\section{Methods}

Clinical procedures. Patients were studied within $48 \mathrm{~h}$ of hospital admission. Initial management comprised full clinical assessment, baseline blood samples for routine laboratory tests, oral or intravenous rehydration and commencement of antimicrobial therapy. The children with enteric fever received oral or parenteral chlor- 